\title{
Evaluation and Endoscopic Management of Esophageal Submucosal Tumor
}

\author{
Weon Jin Ko, Ga Won Song and Joo Young Cho \\ Digestive Disease Center, CHA Bundang Medical Center, CHA University, Seongnam, Korea
}

Submucosal tumors (SMTs) originate from tissues that constitute the submucosal layer and muscularis propria, and are covered by normal mucosa. Esophageal SMTs are rare, accounting for $<1 \%$ of all esophageal tumors. However, the recent widespread use of endoscopy has led to a rapid increase in incidental detection of SMTs in Korea. Esophageal SMTs are benign in $\geq 90 \%$ of cases, but the possibility of malignancies such as gastrointestinal stromal tumor and malignant leiomyosarcoma still exists. Therefore, patients undergo resection in the presence of symptoms or the possibility of a malignant tumor. For resection of esophageal SMTs, surgical resection was the only option available in case of possible malignancy, but minimally invasive surgery by endoscopic resection is becoming more preferable to surgical resection with the development of endoscopic ultrasonography, endoscopic techniques, and other devices. Clin Endosc 2017;50:250-253

Key Words: Submucosal tumor; Endosonography; Endoscopic resection

\section{INTRODUCTION}

The incidence of esophageal submucosal tumors (SMTs) is relatively low, with leiomyoma accounting for majority of the cases (70\% to $80 \%)$. Esophageal leiomyomas are usually detected in people in their 20s to 50s. They are more frequent in men than in women in the ratio $2: 1$, and about $80 \%$ occur in the mid and lower esophagus. ${ }^{1}$ However, these require resection if they cause dysphagia, obstruction, pain, and other symptoms, or if diagnostic testing does not rule out a malignancy. Gastrointestinal stromal tumor (GIST) is a rare disease, accounting for $<5 \%$ of the cases, but accompanies the possibility of a malignancy, even malignant leiomyosarcoma in rare cases. ${ }^{2}$ The exact prevalence is unknown, but a report in

Received: July 27, 2016 Revised: September 25, 2016

Accepted: September 25, 2016

Correspondence: Joo Young Cho

Digestive Disease Center, CHA Bundang Medical Center, CHA University, 59 Yatap-ro, Bundang-gu, Seongnam 13496, Korea

Tel: +82-31-780-5641, Fax: +82-31-780-5005, E-mail: cjy6695@dreamwiz.com

(c) This is an Open Access article distributed under the terms of the Creative Commons Attribution Non-Commercial License (http://creativecommons.org/ licenses/by-nc/3.0) which permits unrestricted non-commercial use, distribution, and reproduction in any medium, provided the original work is properly cited.
Korea states that of 48,926 cases of upper gastrointestinal (GI) endoscopy performed as part of medical examination, SMTs were detected in $1.45 \%$, mostly involving the stomach $(0.89 \%)$ followed by the esophagus $(0.45 \%)^{3}$

\section{DIAGNOSIS OF ESOPHAGEAL SMT}

\section{Endoscopic findings}

Upon detection of an SMT, the first step is to determine whether the tumor is intrinsic or extrinsic to the GI wall. Extrinsic compressions can be caused by either normal structures or tumors of the mediastinum. The normal structures include the aortic arch, left bronchus, and spine. In particular, patients with congenital deformities require differential diagnosis. Moreover, lymph node metastasis of lung cancer, recurrent esophageal cancer, and mediastinal tumor were reported to be the causes of extrinsic compressions. Extrinsic compressions can be characterized by a large mass, often relocating depending on the patient's position or breathing. The lesion is usually identical to the mucosa in terms of color. However, a yellow color implies a granular cell tumor or lipoma; a rather transparent appearance, a duplication cyst or lymphangioma; 
and a blue color, a vascular lesion such as hemangioma and varix. In addition, one recommended technique for differential diagnosis of SMTs is pressing the mass with forceps. A mass originating from the GI wall moves about under the subepithelium (rolling sign) when pressed. A solid, less mobile mass implies a leiomyoma, GIST, or granular cell tumor. Pressing with forceps create a depression into a less solid mass such as a lipoma (pillow sign) or causes fluid contents of a cyst to spread out (cushion sign).

\section{Endoscopic ultrasonography}

Endoscopic ultrasonography (EUS) allows observation of the size, layer of origin, echo pattern, and internal properties of SMTs, and enlarged lymph nodes near them. Layer of origin and echo pattern are particularly useful for differential diagnosis of SMTs. Echo patterns of SMTs appear as hyperechoic, isoechoic, hypoechoic, or anechoic compared with the echo patterns of the surrounding normal organs. A hypoechoic lesion in the muscular layer is most likely a leiomyoma. In the submucosal layer, a hypoechoic pattern implies a granular cell tumor or carcinoid, while a hyperechoic pattern may suggest a lipoma. An anechoic pattern suggests a cyst, lymphangioma, or vascular lesion. For the observation of esophageal SMTs, catheter probe EUS using water or gel is convenient and thus commonly used. ${ }^{4}$ Despite being the most useful technique for the diagnosis of SMTs, EUS still accompanies the risk of interobserver variation depending on observer proficiency. The interobserver agreement was reported to be high for the diagnosis of cystic disease, lipoma, and extrinsic compressions; low for vascular lesion, leiomyoma, and stromal tumor; and very low for carcinoid and metastatic cancer. One limitation of EUS is that it cannot be used as the final diagnostic modality and requires histological confirmation. ${ }^{5}$

\section{Histological examination}

Common biopsy, in which tissues are collected only from the mucosa and part of the submucosa, is not recommended for all SMTs. However, some SMTs (those that originate from the muscularis mucosa layer, such as leiomyomas and granular cell tumors) can be identified by using common biopsy. Lipoma or lymphangioma sometimes can be inferred based on the exposed tissue after biopsy. According to studies on
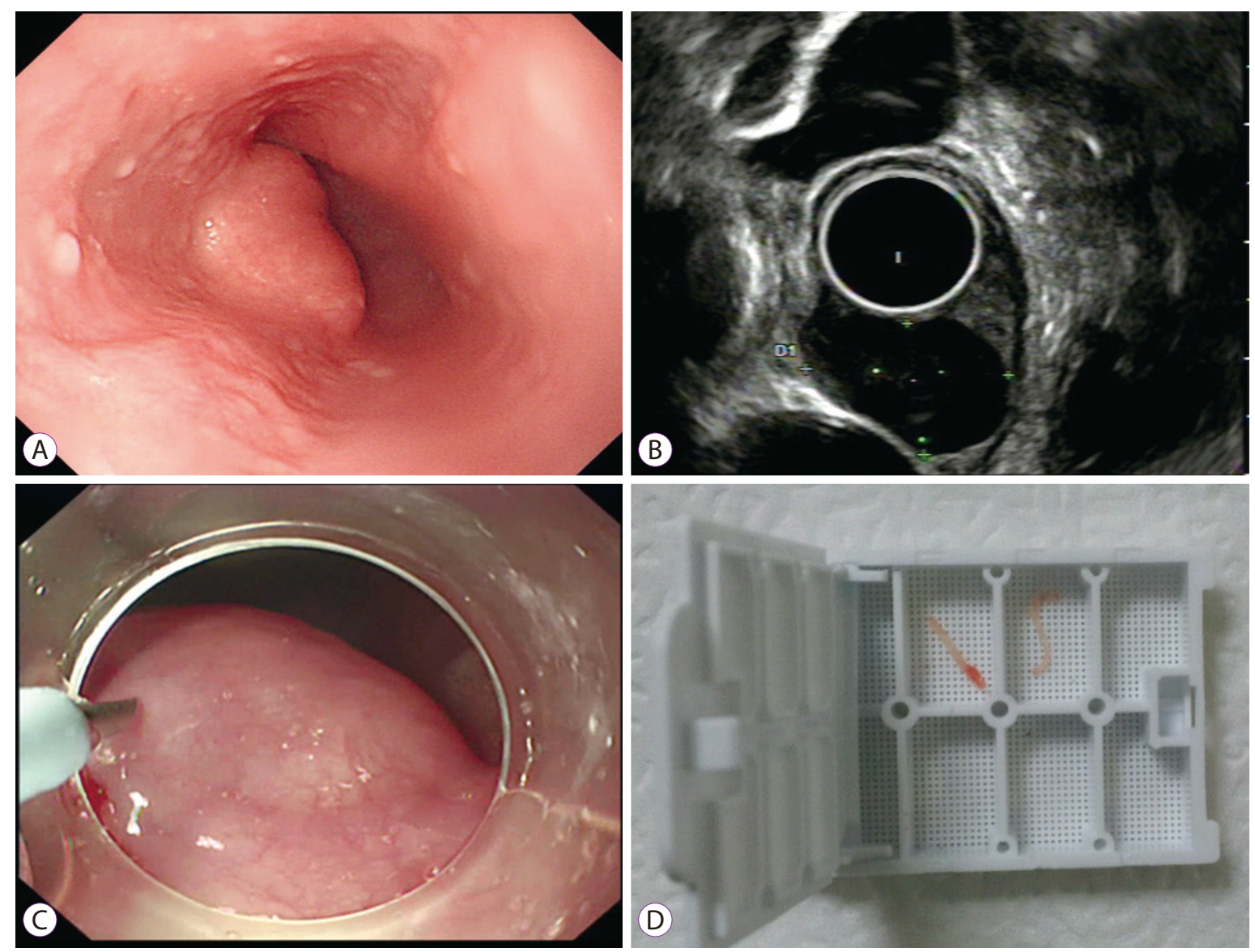

Fig. 1. Tru-Cut biopsy using a flexible endoscope for the diagnosis of submucosal tumor (SMT). (A) SMT observed by using a flexible endoscope. (B) On endoscopic ultrasonography, the SMT is located in the muscularis propria. (C) Tru-Cut biopsy using a flexible endoscope. (D) Specimens. 
SMTs with the likelihood of malignancy, malignancy was observed in $3.7 \%$ of GISTs smaller than $2 \mathrm{~cm},{ }^{6}$ and metastasis was observed in $2 \%$ of carcinoids smaller than $1 \mathrm{~cm} .{ }^{7}$ For this reason, recent guidelines recommend histological diagnosis for SMTs larger than $2 \mathrm{~cm}$ (United States and Europe) or $1 \mathrm{~cm}$ (Japan). However, current biopsy techniques using EUS, such as fine-needle aspiration and Tru-Cut biopsy, have a diagnostic accuracy of $52 \%$ to $86 \%$. Fine-needle aspiration does not provide enough amounts of tissue for differentiation of SMTs, and Tru-Cut biopsy is difficult to handle. Recently, Tru-Cut biopsy using a flexible endoscope instead of EUS was found quite useful for the diagnosis of esophageal SMTs larger than $2 \mathrm{~cm}$ (Fig. 1).

\section{TREATMENT OF ESOPHAGEAL SMT}

The recent widespread use of endoscopic submucosal surgery has made endoscopic diagnosis and treatment of esophageal SMTs possible. Endoscopic submucosal dissection (ESD) allows histological examination of SMTs in the muscularis propria. This is called the endoscopic unroofing technique because it involves incision of the mucosa covering a suspected GIST lesion to confirm the tumor tissue and to obtain biopsy samples with forceps. Endoscopic resection alone is often enough to achieve complete resection of SMTs in the muscular or submucosal layer. However, the histological resection rate is still only approximately $78 \%$ with this technique, which often results in intraoperative tumor rupture and complications such as perforation and hemorrhage in $<5 \%$ of cases. ${ }^{9}$ New techniques include endoscopic submucosal tunnel dissection (ESTD) and endoscopic muscularis dissection (EMD). ESTD consists of 5 stages, which was first introduced for the treatment of esophageal achalasia. First, a submucosal solution is injected about $5 \mathrm{~cm}$ proximal to the lesion and then a $2-\mathrm{cm}$ mucosal incision is made so that the endoscope can be inserted in the submucosal layer. Afterward, the submucosal layer is dissected with a knife to create a submucosal tunnel until reaching about 1 to $2 \mathrm{~cm}$ distal to the SMT. Then, the SMT is resected. Hemorrhage is controlled, and then the proximal

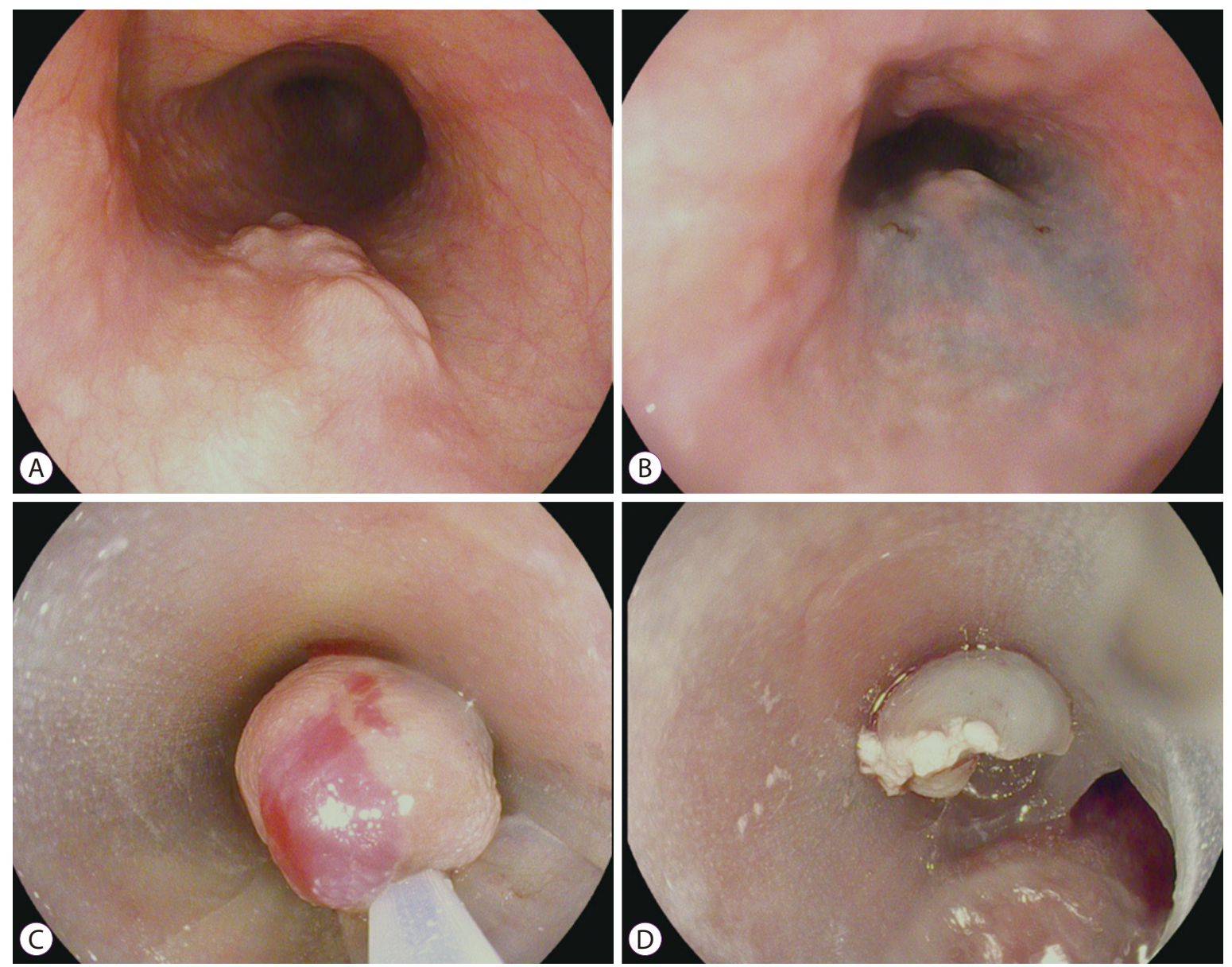

Fig. 2. Application of the three-dimensional printing technique for the side-hole cap during endoscpic mucosal resection with a cap-fitted endoscope applied to a submucosal tumor (SMT). (A) An esophageal SMT. (B) Submucosal injection. (C) Snaring for SMT. (D) Resection of the lesion. 
mucosal incision is closed with clips. EMD differs from ESD in that it requires precutting of the mucosa. The lesion is then dissected by blunt dissection (by pushing between the tumor and muscular layer), without using electrical dissection, to minimize injury to the muscular layer. Afterward, the mucosa is closed as much as possible with endoclips. These methods are useful for removing SMTs $>2 \mathrm{~cm}$ but are complicated. We investigated endoscopic procedures that are easily applicable to smaller tumors. The first one is endoscopic submucosal resection with a side-hole cap, which was developed by using a three-dimensional (3D) printer (Fig. 2). It allows the use of 3D printers for making customized endoscopic accessories. As an endoscope is inserted parallel to the esophagus, we created a hole on the side of the hood attached to the tip of the endoscope to facilitate tumor resection in the esophagus. With this method, we achieved great results in endoscopic resection of SMTs, which suggests a promising possibility for the development of various endoscopic accessories with a 3D printer. ${ }^{10}$ The second method is endoscopic proper muscle resection with an over-the-scope clip (OTSC). In the light of the huge risk of perforation from SMT resection in the muscularis propria, this technique applies OTSC first as a preventive measure against perforation before starting the actual tumor resection, which provided good results for the resection of SMTs smaller than $2 \mathrm{~cm}$. Another possible technique for SMTs that are too large to be retrieved through the mouth is a multidisciplinary procedure that combines the use of an endoscope to resect SMTs $>5 \mathrm{~cm}$ and a thoracoscope to retrieve the lesion and close the wound.

\section{CONCLUSIONS}

With the growing interest in SMTs and the development of endoscopic devices and technology, the diagnosis and treatment of SMTs have made a lot of progress recently. Now, we can safely perform endoscopic resection of tumors smaller than $2 \mathrm{~cm}$, accurately diagnose larger tumors, and even perform endoscopic resection of SMTs originating from the muscularis propria, which in the past, would be surgically removed because of the risk of perforation. With large-scale studies on endoscopic submucosal surgery techniques and preventive measures of complications such as perforation, and with the use of safer and more precise endoscopic devices, more advanced diagnostic and therapeutic techniques would be available for esophageal SMTs.

\section{Conflicts of Interest}

The authors have no financial conflicts of interest.

\section{REFERENCES}

1. Emory TS, Sobin LH, Lukes L, Lee DH, O'Leary TJ. Prognosis of gastrointestinal smooth-muscle (stromal) tumors: dependence on anatomic site. Am J Surg Pathol 1999;23:82-87.

2. Mutrie CJ, Donahue DM, Wain JC, et al. Esophageal leiomyoma: a 40year experience. Ann Thorac Surg 2005;79:1122-1125.

3. Kim MC. Endoscopic incidence of upper gastrointestinal submucosal tumors and endosonographic findings [dissertation]. Suwon: Ajou University School of Medicine; 2008.

4. Ha TI, Kim GH, Eum JS, et al. Catheter probe endoscopic ultrasonography using the jelly-filled method for esophageal subepithelial lesions. Korean J Gastrointest Endosc 2008;36:125-131.

5. Gress F, Schmitt C, Savides T, et al. Interobserver agreement for EUS in the evaluation and diagnosis of submucosal masses. Gastrointest Endosc 2001;53:71-76.

6. Jeong IH, Kim JH, Lee SR, et al. Minimally invasive treatment of gastric gastrointestinal stromal tumors: laparoscopic and endoscopic approach. Surg Laparosc Endosc Percutan Tech 2012;22:244-250.

7. Mani S, Modlin IM, Ballantyne G, Ahlman H, West B. Carcinoids of the rectum. J Am Coll Surg 1994;179:231-248.

8. Ko WJ, Song GW, Hahm KB, et al. Tissue diagnosis of GI subepithelial tumor only through Trucut biopsy under a forward-viewing endoscope: applicability as newer diagnostic modality. Surg Endosc 2016;30:50095014.

9. Fujishiro M, Yahagi N, Nakamura M, et al. Successful outcomes of a novel endoscopic treatment for GI tumors: endoscopic submucosal dissection with a mixture of high-molecular-weight hyaluronic acid, glycerin, and sugar. Gastrointest Endosc 2006;63:243-249.

10. Ko WJ, Song GW, Hong SP, Kwon CI, Hahm KB, Cho JY. Novel 3Dprinting technique for caps to enable tailored therapeutic endoscopy. Dig Endosc 2016;28:131-138. 